\title{
Crecimiento y Caracterización de Recubrimientos Cerámicos con Aplicaciones como Barreras Térmicas
}

\author{
J. GÓMEZ-GARCíA, P. POZA, V. UTRILLA \\ Dpto. de Ciencia e Ingeniería de Materiales. Escuela Superior de Ciencias Experimentales y Tecnología Universidad Rey Juan Carlos \\ C/ Tulipán, s/n. 28933 Móstoles. Madrid
}

\begin{abstract}
La espectroscopía de impedancia electroquímica resulta ser una técnica de ensayo no destructiva prometedora para evaluar el estado y control de calidad de las barreras térmicas. Este trabajo muestra la variación de las propiedades eléctricas de barreras, depositadas por proyección térmica, medidas por espectroscopía de impedancia electroquímica en función de la oxidación isotérmica al aire con diferentes tiempos de exposición. Finalmente se relacionan los resultados de la espectroscopia de impedancia con la microestructura, estudiada mediante microscopía electrónica de barrido ambiental y microanálisis de rayos $X$.
\end{abstract}

Palabras clave: Barreras térmicas, Espectroscopía de impedancia electroquímica, Microscopía electrónica de barrido ambiental

\section{Processing and characterization of ceramic coatings for thermal barrier applications}

Electrochemical impedance spectroscopy is being developed as a non-destructive evaluation technique for life-remain assessment and quality control of thermal barrier coatings. In this investigation, changes in electric properties of coatings deposited by thermal spray were measured by electrochemical impedance spectroscopy as a function of the isothermal oxidation in air at different times. Finally, the results obtained by electrochemical impedance spectroscopy were related to the coating's microstructure studied by environmental scanning electronic microscopy and energy-dispersive $\mathrm{X}$-ray microanalysis.

Keywords: Thermal barrier coatings, Electrochemical impedance spectroscopy, Environmental scanning electron microscopy

\section{INTRODUCCIÓN}

Los recubrimientos de barreras térmicas se usan desde los años 70 y tienen su principal aplicación como protección frente a elevadas temperaturas de trabajo, a los ambientes agresivos y al desgaste. Se usan como escudo térmico en componentes que tengan que soportar altas temperaturas para alcanzar mayores eficiencias de trabajo (1-3). Además de las ventajas económicas, al generar la misma cantidad de contaminación, se reduce la proporción de contaminantes frente a la energía generada, como requiere el Protocolo de Kioto (4).

La proyección térmica es un método rápido, barato y universal que permite crecer un depósito sobre un substrato por solidificación del material de aporte (5). Mediante esta técnica se inyectan partículas, en una llama formada por la combustión de un gas como el acetileno y oxígeno, que se funden total o parcialmente y se aceleran hacia el substrato, donde impactan y se aplastan unas sobre otras, creando el recubrimiento. Para lograr una buena unión entre el substrato y el recubrimiento proyectado se debe asegurar la limpieza adecuada del substrato y un cierto nivel de rugosidad, que faciliten la adhesión del depósito.

Los recubrimientos con aplicaciones en barreras térmicas se pueden considerar como un sistema complejo, en el que se deposita un anclaje metálico sobre un substrato metálico y sobre él, una capa cerámica. Como aislante térmico suele emplearse $\mathrm{ZrO}_{2}$ estabilizada debido a su reducida conductividad térmica $(0,8-1,5 \mathrm{~W} / \mathrm{m} \cdot \mathrm{K})(6)$. Sin embargo, no es posible recubrir directamente un substrato metálico con esta cerámica. Aunque el coeficiente de dilatación térmica (CTE) de la $\mathrm{ZrO}_{2}$ es alto $\left(10 \cdot 10^{-6}{ }^{\circ} \mathrm{C}^{-1}\right)$ en comparación con otras cerámicas, resulta pequeño en comparación con los metales $\left(18 \cdot 10^{-6}{ }^{\circ} \mathrm{C}^{-1}\right.$ y $13,3 \cdot 10^{-6}{ }^{\circ} \mathrm{C}^{-1}$ para AISI 304 y níquel, respectivamente) y podrían aparecer tensiones de cizalla en la intercara durante el procesado y el ciclado térmico que se produce durante la vida en servicio del componente. Estas tensiones en la intercara llegarían a provocar grietas y defectos en el recubrimiento. Para evitar este problema se emplea un recubrimiento metálico intermedio rico en níquel y aluminio, que además protege al componente frente a la oxidación a alta temperatura. Esta capa intermedia aumenta la adhesión del recubrimiento cerámico al proporcionar una superficie rugosa que ancla mecánicamente la capa de circona estabilizada. Por otra parte, la resistencia de esta capa intermedia al ataque químico se debe a la presencia de elementos activos como el aluminio, que forman capas de óxidos (principalmente $\mathrm{Al}_{2} \mathrm{O}_{3}$ ) impidiendo la entrada de oxígeno en la estructura (7).

El uso continuado de estos dispositivos hace necesario desarrollar técnicas de evaluación no destructivas (NDE) que permitan predecir el fallo y la calidad de las barreras durante su vida en servicio. La espectroscopía de impedancia electroquímica (EIS) es una técnica NDE, que parece prometedora para evaluar las barreras térmicas. Se ha utilizado como técnica analítica en innumerables aplicaciones, incluyendo estudios de corrosión (8) y caracterización de baterías (9), recubrimientos cerámicos $(10,11)$, semiconductores 
y sensores. Mediante EIS se determina la impedancia de un material sumergido en un electrolito para un intervalo amplio de frecuencias. La impedancia en los materiales, especialmente en aquellos que no son buenos conductores de la electricidad varía con la frecuencia según las características físicas del material y la naturaleza química de las uniones.

La degradación de las barreras térmicas se produce por su oxidación a alta temperatura, que provoca la aparición de óxidos en la intercara cerámica - capa de enganche o en la propia capa de enganche. Por otra parte, las barreras térmicas pueden fallar por delaminación de las capas que forman la estructura, favorecida por el crecimiento de óxidos frágiles en la intercara. Los óxidos generados durante la vida en servicio de la barrera, principalmente $\mathrm{Al}_{2} \mathrm{O}_{3^{\prime}}$, son en general malos conductores de la electricidad y la aparición de delaminaciones favorece esta baja conductividad. Por tanto, EIS parece una técnica adecuada para evaluar la degradación de las barreras durante su vida en servicio (10-13).

El objetivo de este trabajo es relacionar la evolución de la microestructura de barreras térmicas oxidadas en aire a diferentes tiempos de exposición y estudiadas mediante microscopia electrónica de barrido ambiental (ESEM) y microanálisis de rayos $\mathrm{X}$ (EDX) con los resultados obtenidos mediante EIS. La correlación observada en el espectro de impedancia de recubrimientos en diferentes estados de degradación permitiría caracterizar la degradación de una barrera térmica en servicio mediante EIS.

\section{TÉCNICAS EXPERIMENTALES}

\subsection{Materiales y Proyección}

Se utilizó como substrato acero inoxidable austenítico AISI $304(75 \times 30 \times 5 \mathrm{~mm})$, tratado mecánicamente con una grallanadora (Jet Stream 22) con polvo de corindón (granulometría 20) para eliminar la capa de óxido que pudiera tener e introducir rugosidad superficial para mejorar la adherencia. Se aplicó un recubrimiento metálico Ultrabond 51000 de Ni-6Al-5Mo (\% en peso) y uno cerámico MetaCeram 28085 de $70 \mathrm{ZrO}_{2}-30 \mathrm{CaO}$ ( $\%$ en peso) por proyección térmica de oxiacetileno empleando un equipo de proyección Castolin DS 8000. La pistola de proyección se colocó en un sistema automático en el eje $\mathrm{x}$, y manual en los ejes y y z. Los substratos se colocaron en un sistema de retención para proyectar sobre ellos. Los parámetros de proyección utilizados fueron la velocidad paralela en el eje $x\left(V_{\mathrm{x}}\right)$, la distancia entre la pistola y el substrato $\left(\mathrm{d}_{\mathrm{p}}\right)$, la distancia entre líneas proyectadas $\left(\mathrm{D}_{\mathrm{l}}\right)$, la apertura del contenedor de polvo y el tipo de boquilla. Estos parámetros se determinaron en series de proyección previas hasta fijar los valores recogidos en la tabla I.

Algunos de los recubrimientos proyectados se trataron térmicamente en un horno Carbolite ELF 11/ 6 provocando su oxidación isoterma durante 48 y 140 horas a una temperatura de $400{ }^{\circ} \mathrm{C}$ y durante 48 y 120 horas a una temperatura de 600 ${ }^{\circ} \mathrm{C}$. Las probetas tratadas se enfriaron durante una hora dentro del horno para evitar el choque térmico.

\subsection{Caracterización}

Las probetas se prepararon con una cortadora de disco de diamante a baja velocidad para minimizar el daño mecánico en el recubrimiento. Las probetas cortadas se embutieron en una resina conductora para su posterior desbastado hasta granulometría 1200, pulido con pasta de diamante hasta $3 \mu \mathrm{m}$ y acabado final con una suspensión de alúmina. Finalmente las probetas se introducen en un baño de ultrasonidos con acetona e isopropanol.

Los recubrimientos depositados y las probetas oxidadas se estudiaron en un ESEM Philips XL30 provisto de microanálisis de rayos $X(E D X)$ para analizar semicuantitativamente su microestructura, homogeneidad y la degradación de la barrera térmica durante el proceso de oxidación isotérmica. Se utilizaron el detector de secundarios gaseosos (GSE) y el detector de electrones retrodispersados (BSE) en condiciones ambientales, introduciendo vapor de agua (presión parcial de 0,6 Torr) para evitar efectos de carga en la capa cerámica.

Se eliminó el substrato de acero para analizar únicamente las características eléctricas de los recubrimientos. El anclaje metálico se trató químicamente con decapante para eliminar la capa oxidada y poder colocar uno de los electrodos por soldadura blanca de $40 \% \mathrm{~Pb}-60 \% \mathrm{Sn}$. Las medidas EIS se realizaron en un sistema AUTOLAB mod. PGSTAT 30 con tres electrodos en una celda plana estándar a temperatura ambiente. El electrolito utilizado en estas medidas fue 0,01 $\mathrm{M}\left(\mathrm{K}_{3} \mathrm{Fe}(\mathrm{CN})_{6} / \mathrm{K}_{4} \mathrm{Fe}(\mathrm{CN})_{6} \cdot 3 \mathrm{H}_{2} \mathrm{O}\right)$. El electrodo de referencia y contraelectrodo empleados fueron $\mathrm{Ag} / \mathrm{AgCl}$ y de grafito, respectivamente. Se esperó un tiempo de 15 minutos en cada espectro antes de realizar el ensayo para permitir que se estabilizara el potencial de circuito abierto, además de asegurar la completa penetración del electrolito a través de los poros abiertos. Se tuvo especial cuidado en quitar las burbujas antes de realizar las medidas. Se aplicó una corriente alterna de $10 \mathrm{mV}$ en un intervalo de frecuencias desde $10^{-2}$ hasta $10^{6} \mathrm{~Hz}$. Se tomaron las medidas 3 veces por probeta para establecer un compromiso estadístico. La instrumentación EIS recoge la respuesta de la impedancia, separando la componente real ( $Z^{\prime}$ : resistencia) y la imaginaria ( $Z^{\prime \prime}$ : capacidad o inductancia); representándose en un diagrama de Nyquist (-Z' frente a $\left.Z^{\prime}\right)$.

\section{RESULTADOS Y DISCUSIÓN}

La probeta sin tratar está recubierta por un anclaje metálico del orden de $150 \mu \mathrm{m}$ de espesor y una capa cerámica entorno a $600 \mu \mathrm{m}$. El anclaje (fig. 1) está formado por granos laminares ricos en níquel, aluminio y trazas de molibdeno,

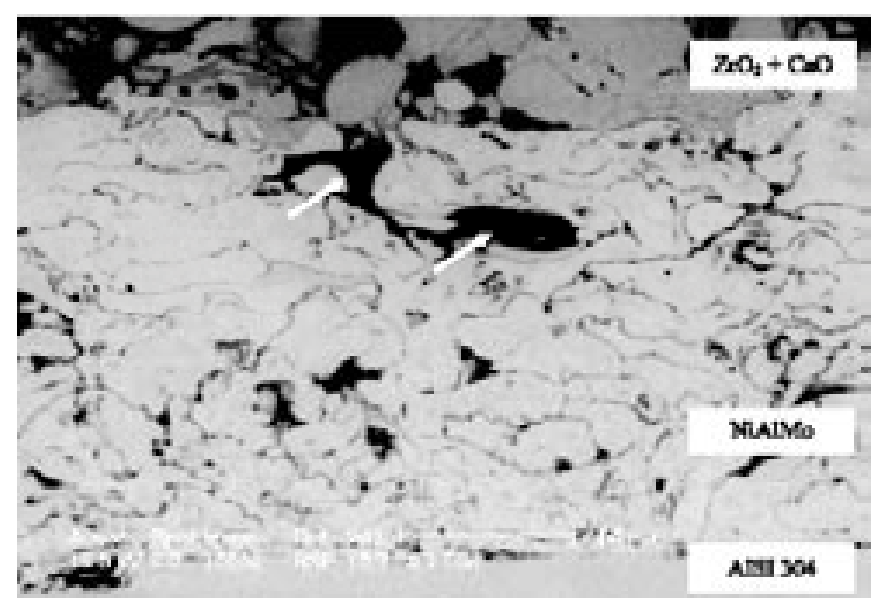

Fig. 1- Imagen de la microestructura de los recubrimientos sin tratar en contraste de electrones retrodispersados. Se encontraron gotas de $\mathrm{Al}_{2} \mathrm{O}_{3}$ en el anclaje metálico (zonas señaladas). 


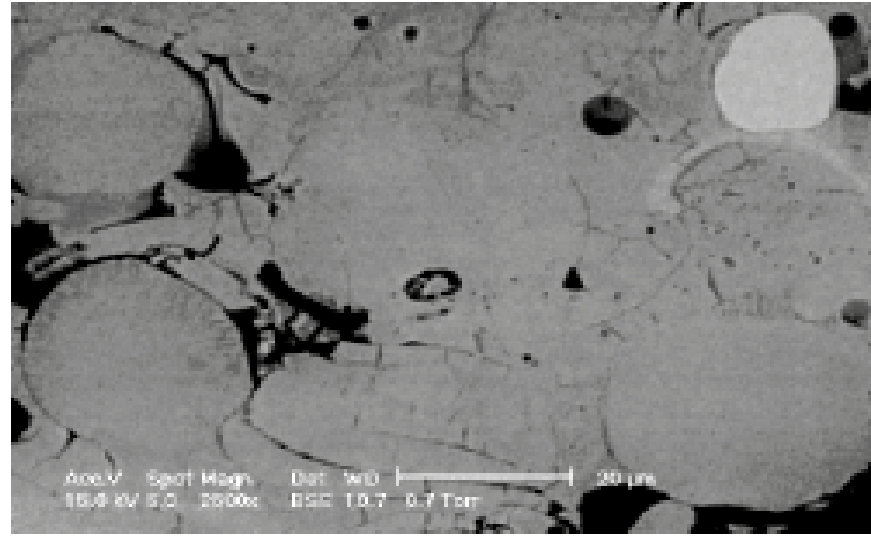

Fig. 2- Detalle de la capa cerámica de la probeta no tratada observado en contraste de electrones retrodispersados. Se observan granos de $\mathrm{ZrO}_{2}-\mathrm{CaO}$ y una zona más clara que contiene itrio.

con poros formados durante la proyección del depósito y zonas ricas en aluminio y oxígeno que rodean los granos de níquel, sugiriendo la formación de alúmina durante la proyección con una llama oxiacetilénica. La capa cerámica (fig. 2) está formada, principalmente, por $\mathrm{ZrO}_{2}-\mathrm{CaO}$ aunque se observaron zonas más claras que contienen trazas de itrio, según EDX. Se observó la porosidad característica del proceso de proyección térmica.

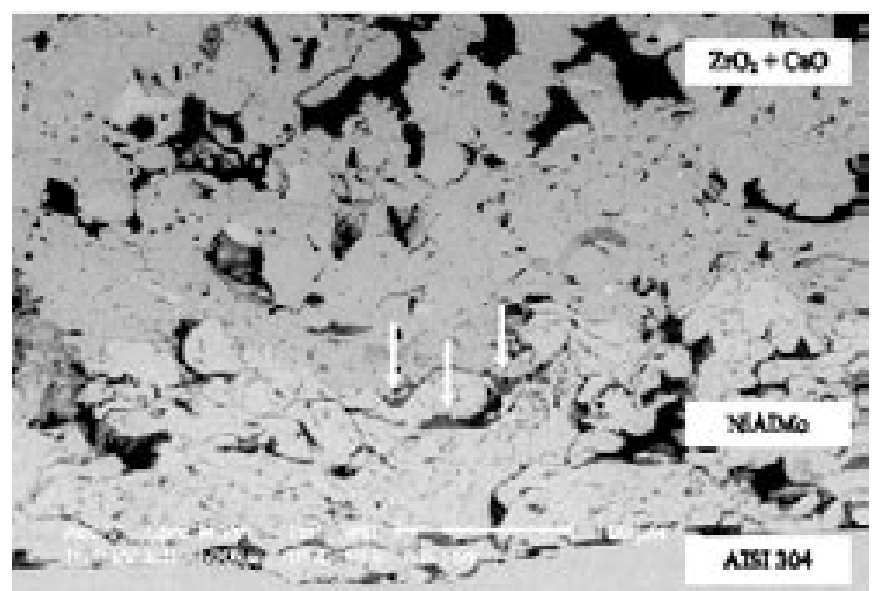

Fig. 3- Imagen de los recubrimientos de la probeta tratada 48 horas a $400{ }^{\circ} \mathrm{C}$ observada en contraste de electrones retrodispersados. Las zonas formadas por $\mathrm{Al}_{2} \mathrm{O}_{3}$ aparecen marcadas en la figura.

Las probetas tratadas térmicamente a $400^{\circ} \mathrm{C}$, prácticamente no se degradaron incluso en el tratamiento durante 140 horas, apareciendo en su microestructura muchas similitudes con la probeta no tratada (fig. 3). Este hecho está de acuerdo con los resultados EIS (fig. 4) que son similares a los encontrados en el material sin tratar, donde se observan

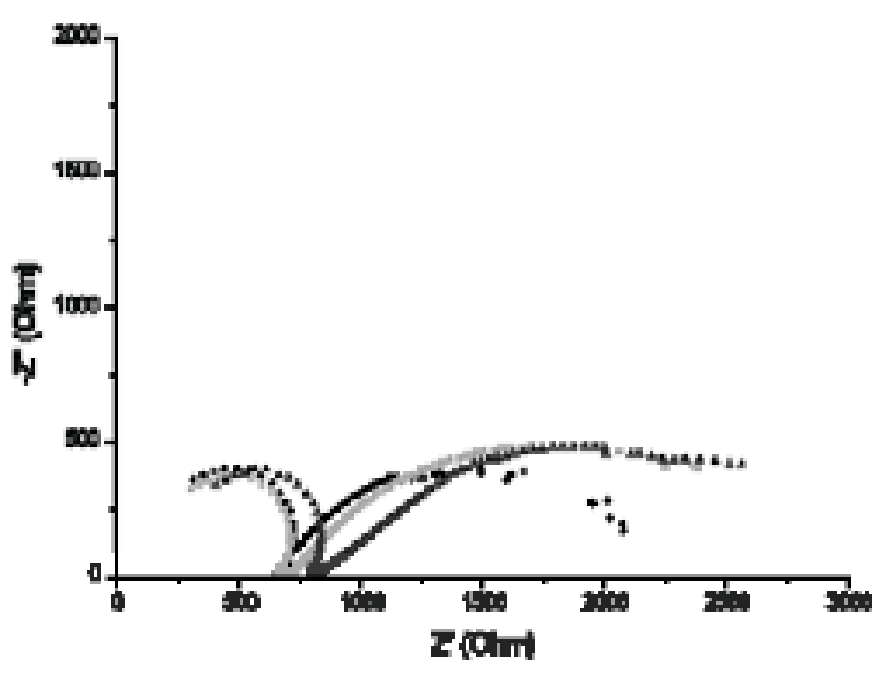

Fig. 4- Diagrama de Nyquist comparativo de las muestras tratadas a $400{ }^{\circ} \mathrm{C}$, durante $48(\square)$ y 140 horas $(\triangle)$, y la no tratada $(\mathbf{j})$. Se observa gran similitud de los espectros, aunque se detecta una pequeña variación para la probeta de mayor tiempo de exposición debido a que comienza su degradación muy lentamente al aparecer $\mathrm{Al}_{2} \mathrm{O}_{3}$ en el anclaje metálico.

dos semicírculos claramente diferenciados. El diámetro del semicírculo de la izquierda (altas frecuencias) asociado a los materiales conductores varía muy poco al aumentar el tiempo de exposición, incluso para las 140 horas de exposición (Tabla II). El diámetro del semicírculo de la derecha (bajas frecuencias), asociado a materiales no conductores, tampoco varía significativamente.

La proporción de zonas ricas en alúmina aumenta en las probetas tratadas térmicamente a $600{ }^{\circ} \mathrm{C}$ (fig. 5). Esto indica que el oxígeno penetró por los poros abiertos en el anclaje provocando su oxidación. Este resultado está de acuerdo con lo observado por Widjaja et all (7) para un anclaje de NiCrCoAlY y una capa cerámica de $\mathrm{ZrO}_{2}$ crecidos por plasma. La oxidación del anclaje NiAlMo es tanto más importante cuanto más largo fue el proceso de oxidación. La oxidación produjo un aumento en la resistencia del recubrimiento y como consecuencia en el diagrama de Nyquist (fig. 6) aumenta el diámetro del semicírculo asociado a altas frecuencias (Tabla II). En la intercara entre el anclaje y el cerámico se produjeron microgrietas que se llenaron de electrolito, dando lugar a un aumento de la resistencia en las medidas EIS, reflejándose en el diámetro del semicírculo asociado a bajas frecuencias. El aumento de las grietas (fig. 7) en la muestra tratada a 120

TABLA II: RESISTENCIA DEL SEMICÍRCULO ASOCIADO A ALTAS FRECUENCIAS PARA LOS DIFERENTES TRATAMIENTOS TÉRMICOS.

\begin{tabular}{|c|c|c|c|c|c|}
\hline Probeta & Sin tratar & $\begin{array}{c}400{ }^{\circ} \mathrm{C}, \\
48 \mathrm{~h}\end{array}$ & $\begin{array}{c}400{ }^{\circ} \mathrm{C}, \\
140 \mathrm{~h}\end{array}$ & $\begin{array}{c}600{ }^{\circ} \mathrm{C}, \\
48 \mathrm{~h}\end{array}$ & $\begin{array}{c}600{ }^{\circ} \mathrm{C}, \\
120 \mathrm{~h}\end{array}$ \\
\hline $\begin{array}{c}\text { Diámetro } \\
(\Omega)\end{array}$ & 450 & 450 & 460 & 640 & 720 \\
\hline
\end{tabular}

TABLA I. PARÁMETROS PARA LA PROYECCIÓN DE LAS DIFERENTES CAPAS

\begin{tabular}{|c|c|c|c|c|c|c|}
\hline Material & $\begin{array}{c}\mathrm{V}_{\mathrm{x}} \\
(\mathrm{mm} / \mathrm{min})\end{array}$ & $\mathrm{N}^{\mathrm{o}}$ pasadas / línea & $\begin{array}{c}\mathrm{D}_{\mathrm{p}} \\
(\mathrm{mm})\end{array}$ & $\begin{array}{c}\mathrm{D}_{1} \\
(\mathrm{~mm})\end{array}$ & Contenedor de Polvo & Aire comprimido \\
\hline Metalceram C28085 & 1500 & 2 & 90 & 3 & $1(\mathrm{ssm} 30)$ & SI (>2 bar) \\
\hline
\end{tabular}




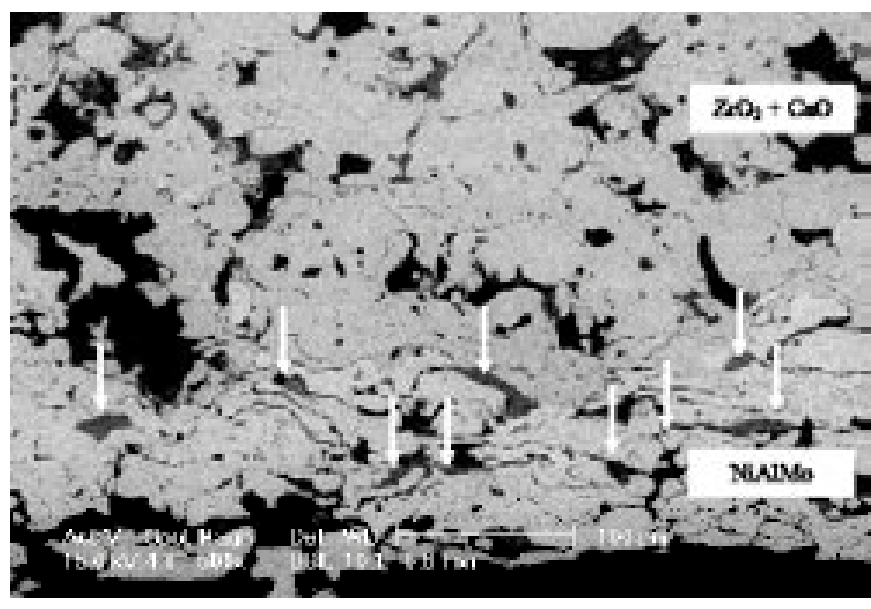

Fig. 5- Imagen de los recubrimientos tratados 120 horas a $600{ }^{\circ} \mathrm{C}$ en contraste de electrones retrodispersados. Se observan los recubrimientos sin el substrato. Se observa un aumento significativo en la proporción de $\mathrm{Al}_{2} \mathrm{O}_{3}$ (zona marcada) con respecto al material sin tratar.

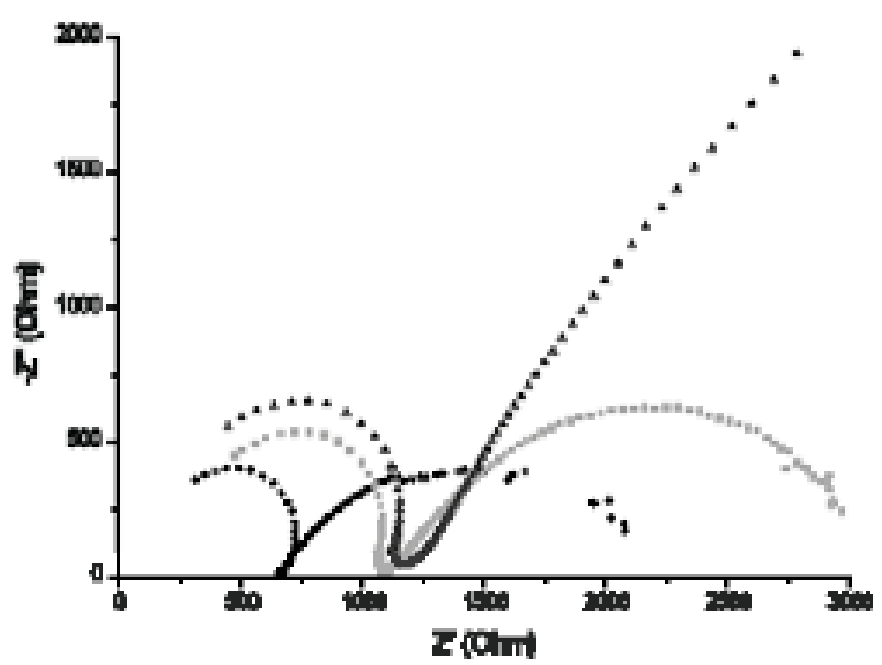

Fig. 6- Diagrama de Nyquist comparativo de las muestras tratadas a $600{ }^{\circ} \mathrm{C}$, durante 48 horas $(\square)$ y 120 horas $(\triangle)$; y la muestra no tratada (j). Se observa como aumenta el diámetro del semicírculo atribuido a materiales conductores, debido al aumento de la alúmina en el anclaje metálico. También observamos la variación en el semicírculo de la capa cerámica debido a las microgrietas producidas que se rellenan de electrolito y por lo tanto, aumenta su resistencia.

horas, provocó una gran variación de este semicírculo. Las microgrietas de las intercaras se produjeron debido a tensiones residuales desarrolladas en la intercara por la diferencia entre coeficientes de expansión térmica del anclaje metálico y los compuestos cerámicos. Este proceso de agrietamiento se agrava por la formación de $\mathrm{Al}_{2} \mathrm{O}_{3}$ en la capa de anclaje con un CTE $\left(8 \cdot 10^{-6}{ }^{\circ} \mathrm{C}^{-1}\right)$ muy inferior al de la capa $\mathrm{ZrO}_{2}-\mathrm{CaO}$.

\section{CONCLUSIONES}

En el anclaje metálico de las barreras térmicas, depositadas por proyección térmica con una llama oxiacetilénica se encontró $\mathrm{Al}_{2} \mathrm{O}_{3}$ entre los granos ricos en níquel. La cantidad de

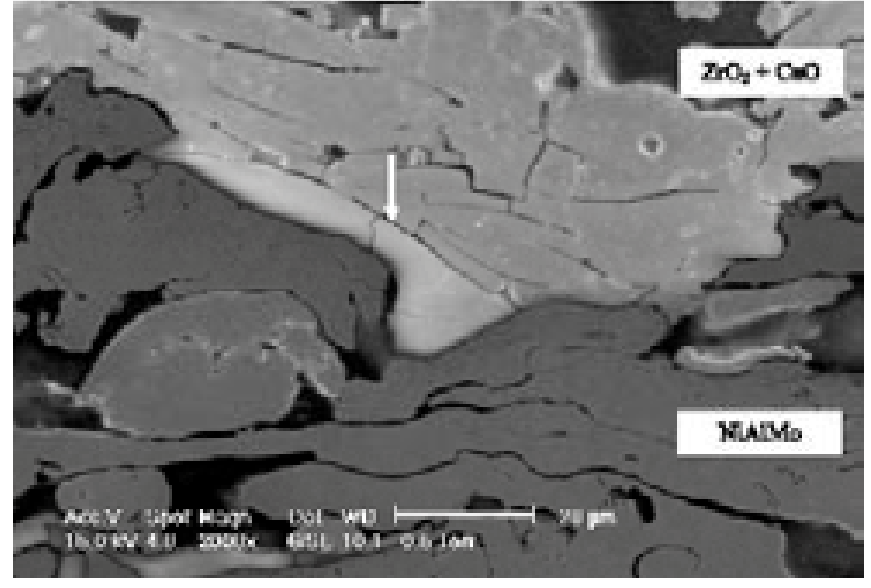

Fig. 7- Detalle de la intercara de la probeta tratada 120 horas a $600{ }^{\circ} \mathrm{C}$ en contraste de electrones secundarios. La flecha indica un grano de $\mathrm{Al}_{2} \mathrm{O}_{3}$ crecido en la intercara. Se observan grietas en la capa cerámica y delaminación en el anclaje metálico.

$\mathrm{Al}_{2} \mathrm{O}_{3}$ presente en las barreras aumentó durante su oxidación isoterma. Este efecto es importante a partir de $600{ }^{\circ} \mathrm{C}$ y aumenta con el tiempo de exposición. Llegaron a observarse microgrietas paralelas al substrato debido a la diferente de CTE en la alúmina y el resto de los elementos.

Los resultados de espectroscopía de impedancia electroquímica reflejan los cambios de la microestructura con una variación de los diámetros de los semicírculos. Por tanto, la espectroscopía de impedancia electroquímica es una técnica no destructiva que permitirá evaluar el estado y la calidad en el servicio de una barrera térmica.

La continuación de este trabajo debe correlacionar el estado de degradación de la barrera con los parámetros obtenidos mediante la espectroscopía de impedancia electroquímica. Para ello, será necesario elaborar modelos de comportamiento que permitan obtener parámetros críticos en la degradación en servicio de la barrera térmica.

\section{AGRADECIMIENTOS}

Los autores agradecen al MCYT (Proyecto MAT 2001-1123C03-03) y a la Universidad Rey Juan Carlos (Proyecto URJC GVC-2003-10) la financiación proporcionada.

\section{BIBLIOGRAFÍA}

1. R.A. Miller, "Thermal Barrier Coatings for Aircraft engines: History and Directions" J. Thermal Spray Technol., [6] 1 35-42 (1997)

2. S. Bose, J. DeMasi-Marcin, "Thermal Barrier Coating Experience in Gas Turbine Engines at Pratt \& Whitney", J. Termal Spray Technol., [6] 199-104 (1997)

3. V. Sergo, D.R. Clarke, "Observation of Subcritical Spall Propagation of a Thermal Barrier Coating" J. Am. Ceram. Soc., [81] 3237-3242 (1998)

4. Naciones Unidas "Protocolo de Kioto" 11 de Diciembre de 1997. Kioto (Japón)

5. V. Higuera, F.J. Belzunce, J.A. Carriles, “Características de los recubrimientos $\mathrm{Cr}_{3} \mathrm{C}_{2}-\mathrm{NiCr}$ en función del sistema de proyección térmica" Bol. Soc. Esp. Ceram. V., 43 [2] $307-310$ (2004)

6. J.C. Diez, J.L. Peña, V.M. Orera, M. Sierra, “Resolidificación con láser de barreras térmicas de circona depositadas por proyección térmica plasma (APS)" Bol. Soc. Esp. Ceram. V., 43 [6] 925-928 (2004)

7. S. Widjaja, A. M. Limarga, T. Hon Yip, "Oxidation Behaviour of a PlasmaSprayed Functionally Graded $\mathrm{ZrO}_{2} / \mathrm{Al}_{2} \mathrm{O}_{3}$ Thermal Barrier Coating", Materials Letters [57] 628-634 (2002) 
8. E. Otero, A. Pardo, M.C. Merino, M.V. Utrilla, M.D. López, J.L. del Peso, "Corrosion Behaviour of IN-800 Superalloy in Waste-Incineration Environments: Hot Corrosion by Molten Chlorides", Ox. Metals [(51) 5-6] 507-525 (1999)

9. P.M. Gomadam, J.W. Weidner, R.A. Dougal, R.E. White, "Mathematical Modeling of Lithium-Ion and Nickel Battery Systems", J. Power Sources 110 [2] 267-284 (2002)

10. X. Wang, J. Mei, P. Xiao, "Determining Oxide Growth in Thermal Barrie Coatings (TBCs) Non-Destructively Using Impedance Spectroscopy", J. Mater. Sci, Lett [20] 47-49 (2001)
11. J. Zhang, V. Desai, "Evaluation of Thickness, Porosity and Pore shape of Plasma Sprayed TBC by Electrochemical impedance spectroscopy", Surf. Coat. Tech [190] 98-109 (2005)

12. B. Jayaraj, S. Vishweswaraiah, V.H. Desai, Y.H. Sohn, "Electrochemical Impedance Spectroscopy of Thermal Barrier Coatings as a Function of Isothermal and Cyclic Thermal Exposure", Surf. Coat. Tech [177-178] 140151 (2004)

13. S. Song, P. Xiao, “An Impedance Spectroscopy Study of High-Temperature Oxidation of Thermal Barrier Coatings", Mat. Sci. Eng. [B97] 46-53 (2003).

Recibido: 01.01 .05

Aceptado: 28.04.05 